\title{
Dialdehyde starch nanoparticles as antitumor drug delivery system: An in vitro, in vivo, and immunohistological evaluation
}

\author{
XIAO SuYao ${ }^{1}$, LIU XuanMing ${ }^{2 *}$, TONG ChunYi ${ }^{2}$, ZHAO LiChao $^{1}$, LIU XiaoJuan $^{1}$, \\ ZHOU AiMei ${ }^{1} \&$ CAO Yong ${ }^{1 *}$ \\ ${ }^{1}$ College of Food Science, South China Agricultural University, Guangzhou 510642, China; \\ ${ }^{2}$ Institute of Life Science and Biotechnology, Hunan University, Changsha 410082, China
}

Received January 3, 2012; accepted February 28, 2012; published online July 4, 2012

\begin{abstract}
Sustaining the release of therapeutic nanoparticles in a cell-, tissue-, or disease-specific manner is a potentially powerful technology. A new drug carrier-dialdehyde starch nanoparticle (DASNP) that can sustain the loading and release of 5-fluorouracil (5-Fu) antitumor drug is reported in this study. IR spectrophotometer and ${ }^{1} \mathrm{H}$ NMR confirmed the formation of aldehyde groups, and scan electron microscope determinations showed that the dialdehyde starch nanoparticles obtained had an average diameter of $90 \mathrm{~nm}$. 5-Fu, the model drug, was conjugated into nanoparticles by aldehyde groups. These 5-Fu-binding nanoparticles significantly enhanced breast cancer cell (MCF-7) inhibition in vitro compared with free 5-Fu. After subcutaneous 0 injection in the breast tumor-loaded rats, 5-Fu-DASNP exhibited remarkable tumor-inhibitory efficacy determined by measuring tumor weight in vivo. The tumor inhibition of 5 -Fu-DASNP was $61 \% \pm 6 \%$, whereas that of free 5 -Fu was only $42 \% \pm 4 \%$. Bcl-2/Bax immunohistochemistry studies indicated that 5-Fu-DASNP remarkably induced tumor tissue necrosis. These results demonstrated that the DASNP prepared in this work is a potentially effective drug carrier.
\end{abstract}

DASNP, 5-Fu, sustaining release, tumor inhibition

Citation: Xiao S Y, Liu X M, Tong C Y, et al. Dialdehyde starch nanoparticles as antitumor drug delivery system: An in vitro, in vivo, and immunohistological evaluation. Chin Sci Bull, 2012, 57: 3226-3232, doi: 10.1007/s11434-012-5342-5

Dialdehyde starch (DAS), one of the most valuable forms of oxidized starch, is prepared by oxidation of starch with periodic acid, a selective oxidant that can cleave the $\mathrm{C} 2-\mathrm{C} 3$ bond of the anhydroglucose units forming the dialdehyde groups [1]. Dialdehyde starch can react with some compounds, such as hydrazine, acid, amine, and imine, through two aldehyde groups in each glucose residual [2]. Thus, it has found wide application in industrial and pharmaceutical fields [3-6]. Majority of DAS production is consumed by the paper industry as wet strength improver [7-9]. DAS also evokes considerable interest in the food industry because of its potential applications, especially as component of biodegradable plastics for packaging, superabsorbents, and traps for removing heavy metals from industrial wastes [10,11].

In pharmaceutics, dialdehyde starch has been studied as a

*Corresponding authors (email: xm105@126.com; caoyong2181@ scau.edu.cn) drug carrier, using its conjugation with isoniazid. The characteristics of dialdehyde starch as a polymer, particularly molecular weight distribution, dissolution, and degradation behavior, have been investigated in vitro in detail $[12,13]$. Dialdehyde starch as nanoparticles have been prepared for use as drug carriers. The results show that dialdehyde starch nanoparticles (DASNP) can be used as antitumor drug carriers, and that they have no acute-cytotoxicity. Hence, they are potentially useful drug carriers [14].

In this study, DASNP was conjugated with 5-Fu to form multiple nanoparticles of 5-Fu-DASNP. 5-Fu-DASNP was characterized in terms of size, IR spectrum, ${ }^{1} \mathrm{H}$ NMR, and drug loading and release. The in vitro anti-cancer activity of 5-Fu-DASNP was determined using an MCF-7 cell line with MTT assay. On the other hand, the in vivo antitumor activity was assessed through a single subcutaneous injection of the nanoparticle formulation into an animal tumor 
model to observe the sustained release effect and to compare it with that of the injection of untreated $5-\mathrm{Fu} . \mathrm{H} \& \mathrm{E}$ staining and $\mathrm{Bcl}-2 / \mathrm{Bax}$ immunohistochemistry studies of the slips of tumors were administered to evaluate the apoptosis of the tumor cells in the body.

\section{Materials and methods}

\subsection{Materials}

5-Fu was obtained from Sigma (Missouri, USA). All cell culture plasticwares were purchased from Corning (NY, USA). The RPMI 1640 cell culture medium and phosphate buffered saline (PBS) were obtained from Gibco (Grand island, NY, USA).

\subsection{Synthesis of DASNP and 5-Fu-DASNP}

DASNP was prepared according to our previously published paper [14]. Hydrolyzing starch was oxidized with sodium periodate $\left(\mathrm{NaIO}_{4}\right)$ to obtain dialdehyde starch (DAS). DAS was cross-linked with $\mathrm{POCl}_{3}$ in water-in-oil micro-emulsion to obtain dialdehyde starch nanoparticles (DASNPs) under room temperature. The oxidation degree (OD) of the prepared DASNPs was $40 \% \pm 5 \%$, and the diameter was in the range of 80 to $100 \mathrm{~nm}$, which were uniform in size and well dispersed.

About $50 \mathrm{mg}$ DASNP was suspended in $1 \mathrm{~mL}$ distilled water. The $\mathrm{pH}$ was adjusted from 3 to 5 using $1 \mathrm{~mol} / \mathrm{L}$ of $\mathrm{HCl}$. 5-Fu dissolved in DMSO was added to the aqueous solution. The mixed solution was incubated for $3 \mathrm{~h}$ under room temperature (about $25^{\circ} \mathrm{C}$ ). The resultant product was separated with ethanol and freeze dried. The product was completely dissolved in hot water. UV spectrophotometer (UV1600, Beijing Rayleigh Analytical Instrument Corp., China) was used to check the 5-Fu content in the solution, and pure 5-Fu was used to construct a calibration curve.

\subsection{Characteristics of DASNP and 5-Fu-DASNP}

Solid samples of DASNP and 5-Fu-DASNP ( 200 mg) were ground with potassium bromide and then compressed into a thin pellet using a Model B Carver Laboratory Press (Fred S. Carver Inc., NJ, USA). The samples were analyzed using an infrared spectrophotometer (WQF-410, RAYLEIGH ${ }^{\mathrm{TM}}$, Beijing Rayleigh Analytical Instrument Corp., China). The ${ }^{1} \mathrm{H}$ NMR spectrum of 5-Fu-DASNP was verified using the Nucleus Magnetic Resonance system (INOVA-400, VARIAN ${ }^{\mathrm{TM}}$, Varian Inc., USA). A scanning electron microscope (SEM) (JSM-5800, JEOL Corp., Japan) was used to check the appearance and diameters of DASNP and 5-Fu-DASNP.

\subsection{Drug release rate determination}

About $10 \mathrm{mg}$ 5-Fu-DASNP was suspended in $5 \mathrm{~mL}$ PBS at
$\mathrm{pH}$ 7.4. The solution was dialyzed against a $50 \mathrm{~mL}$ buffer of a different $\mathrm{pH}$ value under stirring at $100 \mathrm{r} / \mathrm{min}$ and $37^{\circ} \mathrm{C}$. About $5 \mathrm{~mL}$ of the medium was removed at certain intervals and replaced with fresh buffer to maintain the total volume. The amount of 5-Fu in the dialyzed solution was analyzed using UV-spectroscopy (UV1600, Beijing Rayleigh Analytical Instrument Corp., China). DASNP (without drug) was used as the blank sample, and the calibration curve was constructed as above.

\section{5 vitro cytotoxicity assay}

The cytotoxicity of 5-Fu, DASNP, 5-Fu/DASNP, and 5-FuDASN were determined by measuring the cell growth inhibition of the nanoparticles using a micro-reader (MK3, Thermo Electron Corp., USA). In this standardized assay, opaque white 96-well microtiter tissue culture plates were seeded with MCF-7 cells at a density of 10000 cells per well in $100 \mu \mathrm{L}$ RPMI 1640 complete medium containing $10 \%$ fetal bovine serum [15]. The cells were acclimated for $24 \mathrm{~h}$. The medium was removed, and $100 \mu \mathrm{L}$ of the fresh medium containing the test compounds was added. All compounds were initially solubilized in $100 \%$ DMSO up to a final DMSO concentration of $1.0 \%$ each. Each of the compound concentration was tested in triplicate.

\subsection{In vivo antitumor activity assay}

The antitumor activities against subcutaneously implanted solid tumor induced by human breast cancer cells in nude mice were evaluated for saline, DASNP, 5-Fu, and 5-FuDASNP. BALB/c female nude mice (6 weeks old) were divided into 4 different groups consisting of 6 mice in each group [15]. MCF-7 cells $\left(2 \times 10^{6}\right.$ cells in $0.1 \mathrm{~mL}$ PBS $(\mathrm{pH}$ 7.4)) were subcutaneously transplanted along the flank of the mice on day 0 . The peri-tumoral drug injection started on day 8 when the tumor diameter reached approximately $220 \mathrm{~mm}^{3}$. The first group (control group) was injected with $0.1 \mathrm{~mL}$ saline every other day over the following $10 \mathrm{~d}$, whereas the second group was injected with DASNP (20 $\mathrm{mg} / \mathrm{kg}$ body weight) the same way. The rest of the groups of mice were injected with $20 \mathrm{mg} / \mathrm{kg}$ body weight of free $5-\mathrm{Fu}$ (third group), and with $20 \mathrm{mg} / \mathrm{kg}$ body weight of $5-\mathrm{Fu}-$ DASNP (fourth group). The drug was injected six times. The tumor diameters in the mice were monitored using a digital caliper. These diameters were used as index for the in vivo antitumor activity of the drug. When the experiment was concluded after injection on day 10 , the mice were sacrificed, and the solid tumors were obtained and weighed. The inhibitory ratio of the tumor was calculated using the formula $\left(1-W_{\text {drug }} / W_{\text {saline }}\right) \times 100 \%$, where $W$ is the weight.

\subsection{Immunohistochemistry assay}

$B c l-2$ is an inhibiting apoptosis gene largely expressed in 
active tumor cells, whereas Bax is an opposite gene that promotes apoptosis. Both expressions compete in the tumor tissue. Thus, the ratio of Bcl-2-to-Bax was used to evaluate the apoptosis of tumor cells [16,17]. Immunohistochemical methods were adopted in this research. Polyclonal rabbit anti-rat/mouse Bcl-2 antibody (Boster ${ }^{\mathrm{TM}}, \mathrm{CHN}$, at a dilution ratio of 1:60) and polyclonal rabbit anti-rat/mouse Bax antibody (Boster ${ }^{\mathrm{TM}}$, CHN, at a dilution ratio of $1: 40$ ) were used as primary antibodies. Paraffin-embedded tumor tissue sections were used for Bcl-2/Bax staining. The deparaffinized and rehydrated sections were treated through microwave irradiation twice at $550 \mathrm{~W}$ with $10 \mathrm{mmol} / \mathrm{L}$ citrate buffer (pH 6) for 3 min. After blocking with $4 \%$ normal goat serum, the sections were incubated for $1 \mathrm{~h}$ at room temperature with polyclonal rabbit anti-rat/mouse Bcl-2 antibody at a dilution ratio of 1:60 in PBS (or with polyclonal rabbit anti-rat/mouse Bax antibody at a dilution of 1:40 in PBS) as primary antibodies, then with goat-biotinylated anti-mouse IgG, and finally with avidin-biotin complex containing horseradish peroxides [18]. Immunostaining was detected with diaminobenzidine, and the slips of the tissues were counterstained with hematoxylin [19]. All slips were photographed using an inverted fluorescence microscope (TE-2000U, Nikon, Japan).

\section{Results}

\subsection{Conjugation of 5-Fu to DASNP}

5-Fu has an imine group that can be used for conjugation to DASNP. The concentration of 5-Fu in 5-Fu-DASNP was $10 \% \pm 2 \%$, as detected by a UV spectrophotometer. The SEM image showed that the drug loading nanoparticles were uniform in size, between 100 and $120 \mathrm{~nm}$, and well dispersed (Figure 1). Compared with the starch curve, a peak of $1735 \mathrm{~cm}^{-1}$ appeared in the curve of DASNP, which is the band most characteristic of $\mathrm{C}=\mathrm{O}$ vibrations in aldehyde groups. In the 5-Fu-DASNP curve, an acute-peak of $2931 / 2890 \mathrm{~cm}^{-1}$ was replaced with the $2925 \mathrm{~cm}^{-1}$ peak, and
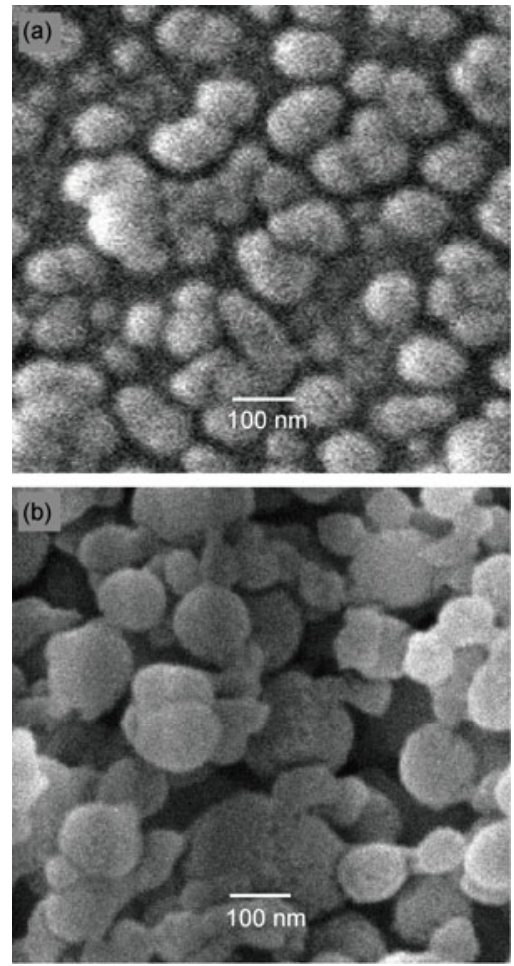

Figure 1 SEM images of DASNP (a) and 5-Fu-DASNP (b).

the 1732 and $779 \mathrm{~cm}^{-1}$ peaks disappeared. However, the $3253(-\mathrm{N}-\mathrm{H}), 1687(-\mathrm{CO}-\mathrm{N}-), 1248(-\mathrm{C}-\mathrm{N}-)$, and 1155 $\mathrm{cm}^{-1}(-\mathrm{C}-\mathrm{F})$ peaks appeared in the 5-Fu-DASNP IR spectrum (Figure 2). In the ${ }^{1} \mathrm{H}$ NMR spectrum, resonance peaks of chemical displacements $\delta=6.0 \times 10^{-6}(-\mathrm{CH}-\mathrm{N}-)$ and $\delta=7.4 \times 10^{-6}(-\mathrm{CH}-\mathrm{O}-)$ appeared. These results indicate that the conjugation of 5-Fu to DASNP was achieved. In the DASNP curve, the $2931 / 2890 \mathrm{~cm}^{-1}$ double peaks and the $1732 \mathrm{~cm}^{-1}$ peak are characteristic peaks of $\mathrm{C}=\mathrm{O}$ in $-\mathrm{CHO}$, whereas the $779 \mathrm{~cm}^{-1}$ peak is the characteristic peak of $\mathrm{C}-\mathrm{H}$ in -CHO. In the 5-Fu-DASNP curve, the $3253(-\mathrm{N}-\mathrm{H})$, $1687(-\mathrm{CO}-\mathrm{N}-), 1248(-\mathrm{C}-\mathrm{N}-)$, and $1155 \mathrm{~cm}^{-1}(-\mathrm{C}-\mathrm{F})$ peaks are apparent.

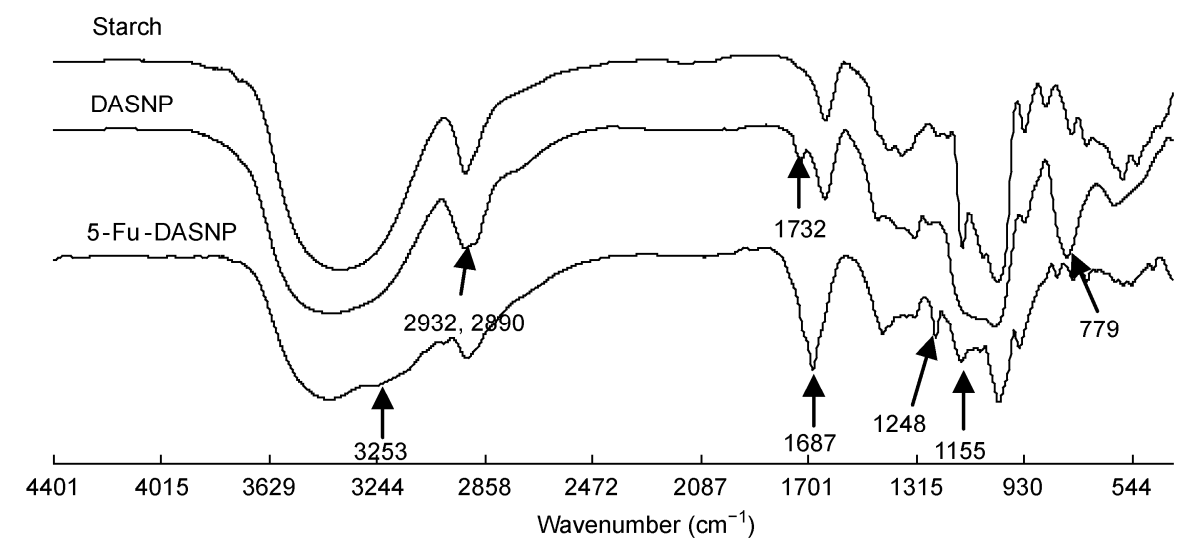

Figure 2 IR spectrogram of the nanoparticles. 


\subsection{Release behavior of 5-Fu from 5-Fu-DASNP}

Figure 3 shows the release profiles of $5-\mathrm{Fu}$ from $5-\mathrm{Fu}-$ DASNP in aqueous solution of different $\mathrm{pH}$ values. The free 5-Fu had a large burst release in PBS (pH 7.4). Its short release duration is attributed to the drug release that occurred with a simple diffusion process through a dialysis bag (M.W. cutoff: 10000, Spectra/Por). 5-Fu release from the nanoparticles was slower in acid solution than in alkaline solution. The half-releasing time $t_{1 / 2}$ was $40 \mathrm{~h}$ at $\mathrm{pH} 3$, $24 \mathrm{~h}$ at $\mathrm{pH} \mathrm{5,16} \mathrm{h}$ at $\mathrm{pH} 7$, and $7 \mathrm{~h}$ at $\mathrm{pH}$ 9. These findings indicate that in faint acid solution, the release of 5-FuDASNP is slow and sustained. The sustained release effect under acidic conditions may be ascribed to DAS, which does not dissolve in acidic solution [20], and to the drug which is released with slow degradation of DASNP. However, DASNP can be dissolved and degraded under alkaline conditions [19]. Thus, no sustaining release effect in the alkaline solution was observed.

\subsection{Tumor cellular cytotoxicity assays (MTT assays) in vitro}

5-Fu, DASNP, 5-Fu/DASNP, and 5-Fu-DASNP were incubated with MCF-7 cells in a medium for $48 \mathrm{~h}$ before the measurement of cell inhibiting rate through MTT assay. The cytotoxicity of the single DASNP was slight (Figure 4), and a high 5-Fu-DASNP inhibiting ratio was observed at low concentrations $(100,200$, and $500 \mu \mathrm{g} / \mathrm{mL})$, and a significant difference in inhibition effect was observed between the 5-Fu-DASNP group and the 5-Fu/DASNP group (Figure 5). These findings demonstrate that the sustained release effect of 5-Fu-DASNP is obvious in vitro. However, under high

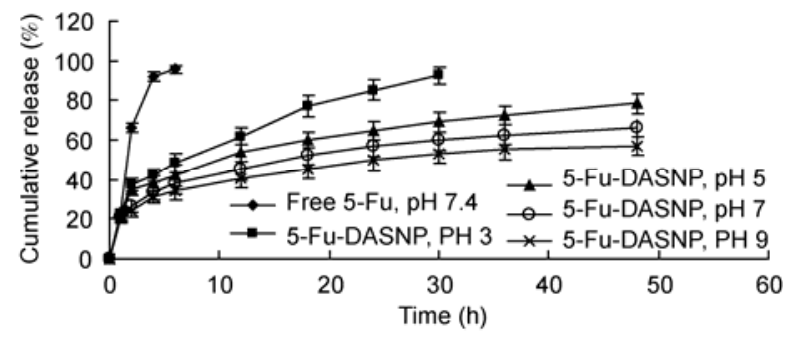

Figure 3 Release rate of 5-Fu-DAS in different $\mathrm{pH}$ solutions.

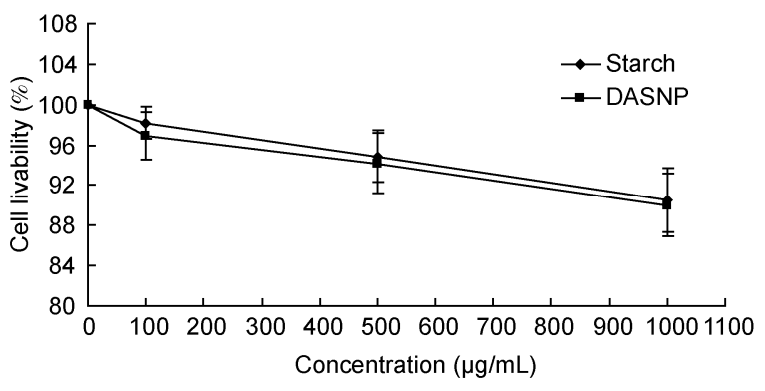

Figure 4 Cellular cytotoxicity assay of DASNP. concentrations $(1000 \mu \mathrm{g} / \mathrm{mL})$, the difference between $5-\mathrm{Fu} /$ DASNP and 5-Fu-DASNP disappeared. Saline was used as the control in this experiment.

\subsection{Efficacy and toxicity studies using human breast cancer MCF-7 in vivo}

To assess the antitumor activity in vivo, nanoparticles containing 5-Fu were directly injected into the surrounding tissues of the subcutaneously implanted solid tumor in mice, and the size of the tumor monitored using a digital caliper. At the end of the experiment, the mice were sacrificed, the solid tumors were obtained, and the inhibiting ratio was calculated. The curves of the tumor volume (Figure 6(a)) showed that the tumor growth trend is controlled by $5-\mathrm{Fu}$ and 5-Fu-DASNP. Compared with saline and DASNP, the controlling effect of 5-Fu-DASNP was the best. The inhibiting ratio was monitored through the final tumor weight, which indicates that 5-Fu-DASNP exhibits obvious effects on the inhibition of tumor growth (Figure 6(b)). 5-Fu exhibited this inhibiting function, but it was weaker than that of 5-Fu-DASNP, whereas a single-shot of DASNP showed very limited effect on antitumor activity (Figure 7).

\subsection{Immunohistochemistry assay}

The tumors were evaluated through hematoxylin and eosin stainings marked with karyon of cells with smalt color. To assess the impact of drug-loading nanoparticles on tumor cell viability, the solid tumors were sliced and the slips were stained with hematoxylin and eosin $72 \mathrm{~h}$ after treatment. They were then examined at low magnification $(\times 100)$. Obvious blanks in the tumor slips of the 5-Fu-DASNP and 5-Fu groups were discovered (Figure 8, upper images), indicating tumor cell death. However, the activity of the 5-Fu-DASNP group was more remarkable than that of the free drug group. No indication of dead cells was observed in the saline and DASNP groups.

Immunohistochemical studies of Bcl-2 and Bax were performed. the results showed significant variant expressions

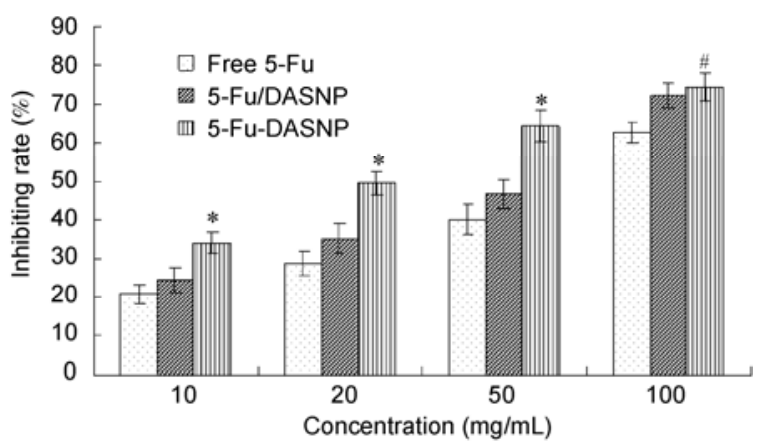

Figure 5 Anti-tumor activities of NPs in vitro. Equivalent concentration of 5-Fu was $10 \mu \mathrm{g} / \mathrm{mL}, 20 \mu \mathrm{g} / \mathrm{mL}, 50 \mu \mathrm{g} / \mathrm{mL}, 100 \mu \mathrm{g} / \mathrm{mL}$. * $P<0.01, n=3$; $\# P>0.05, n=3$. 

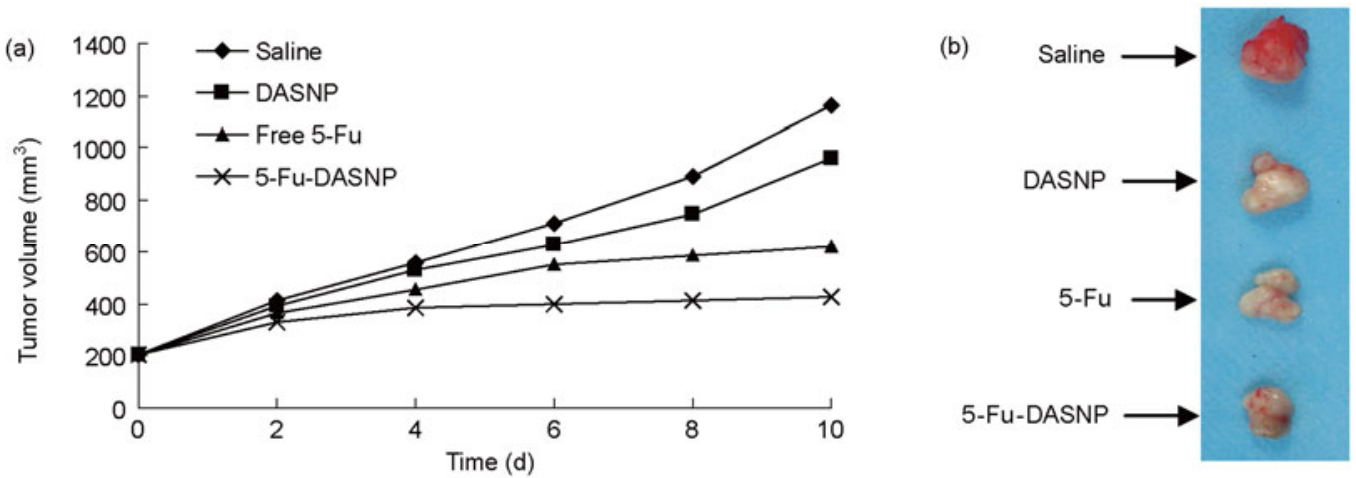

Figure 6 Trend of tumor inhibition in vivo. (a) Tumor growth trend curves; (b) solid tumor images.

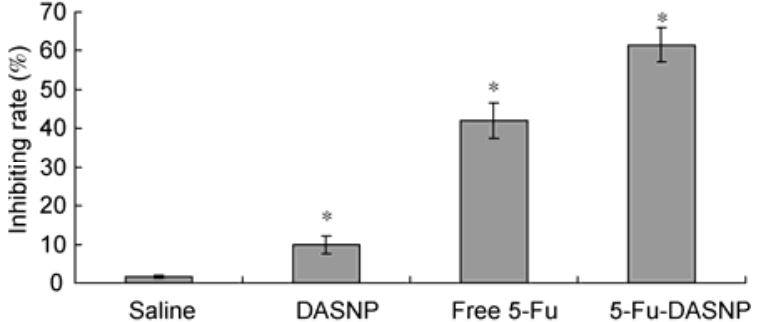

Figure 7 Drug-reduced efficacy for tumor. Tumor weight inhibiting rate, mean+SEM, $n=6 . * P<0.05, n=6$.

between Bcl-2 and Bax in each experiment group (Figure 8, middle and lower images). In the tumor tissue treated with saline, many brown blocks appeared in Bcl-2 staining (Figure 8, middle images), but none was found in Bax staining (Figure 8, lower images). This finding indicates high expression of Bcl-2 in the cytoplasm of the tumor cells and near absence of Bax expression, as well as the absence of apoptosis in the saline group. However, Bcl-2 was faint and Bax was visible in the tumor tissues treated with $5-\mathrm{Fu}$ and 5-Fu-DASNP. The 5-Fu-DASNP expression was most prominent. In the 5-Fu-DASNP group, the Bcl-2 expression was very weak, whereas that of Bax was very strong. These results indicate that drug-loading nanoparticles greatly induce the apoptosis of the tumor tissue cells in vivo.

\section{Discussion}

Sodium periodate can oxidize starch, specifically yielding 2,3-dialdehyde starch. Drug-loading is low when aldehyde content is very low; if the content is very high, the formation of particle drug carriers is difficult [6,20]. Thus, dialdehyde starch nanoparticle with about $40 \%$ of the aldehyde

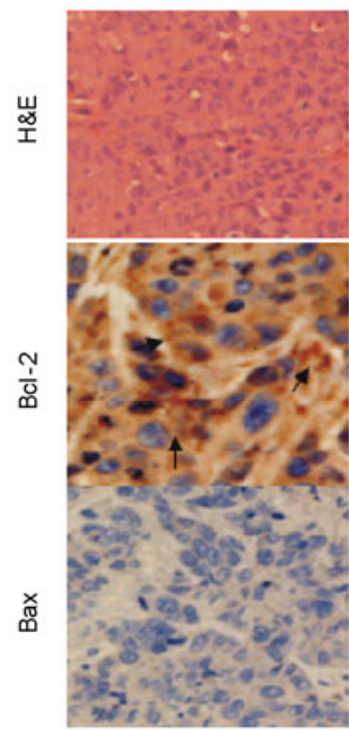

Saline

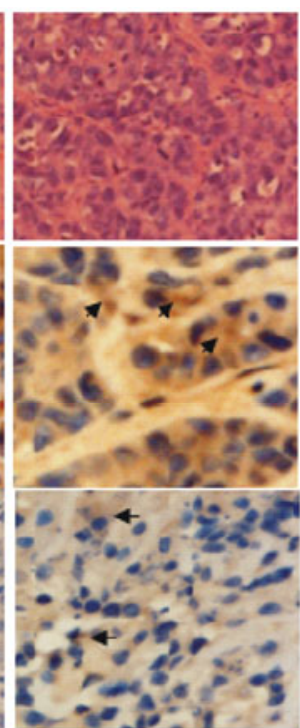

DASNP

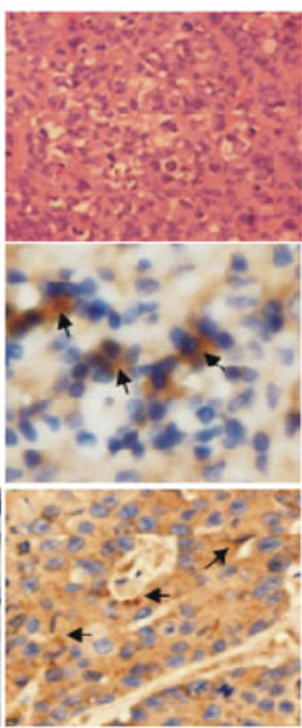

$5-\mathrm{Fu}$

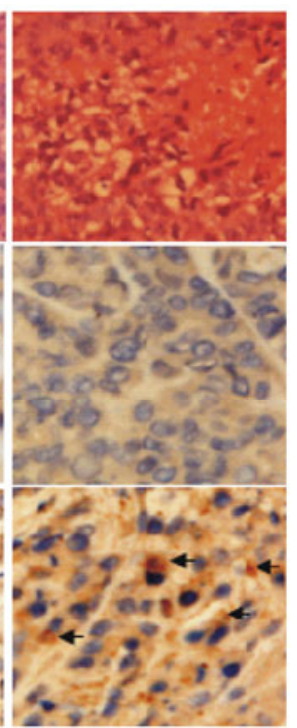

5-Fu-DASNP

Figure 8 H\&E staining and Bcl-2/Bax immunohistochemistry studies of tumors in the saline group, DASNP without drug, 5-Fu, and 5-Fu-DASNP. Upper images are $\mathrm{H} \& \mathrm{E}$ staining of representative specimens at $\times 100$ magnification. Middle images are Bcl-2 staining for each group at $\times 150$ magnification. Lower images are Bax staining for each group at $\times 150$ magnification. The blown yields of Bcl-2 and Bax staining are highlighted in the images (middle and lower). 
group was used, the mass ratio of starch-to-sodium-periodate was controlled, and the reaction time was determined [21]. The cellular cytotoxicity of topical DASNP was similar to starch (Figure 4). Hence, it can be used as a safe drug carrier.

Some protocols have been implemented to improve the curative effect of 5-Fu in tumor therapy, such as preparing prodrugs [22,23] and loading them to polymers [24-26]. However, most of the polymer carriers are physical loading drugs, and the stability of their drug complexes is poor. The chemical activity of DASNP is due to the two aldehyde groups in each glucose monomer, which can conjugate with the imine groups in 5-Fu. The drug release profile (Figure 3) shows that the 5-Fu complex became stable with the sustained release of 5-Fu. An abrupt release occurred during the first one hour of the release profile, which indicates the release of nonspecific physically adsorbed 5-Fu.

Previous studies have indicated that DAS and its derivatives have potential biological activities, such as antineoplastic, antiviral, and anti-inflammatory activities [27]. In the present research, DASNP exhibited tumor growthinhibitory effects on human breast cancer in vitro and in vivo (Figure 7). The flow cytometry results showed that the cell cycle phases of the Mcf-7 cells were altered (Figure 9). A significant increase in $G_{0} / G_{1}$ population and a remarkable reduction in $S$ population were induced in $\mathrm{Mcf}-7$ cells treated with DASNP, compared with the control group. Hence, topical DASNP has some antineoplastic activity through $G_{0} / G_{1}$ phase blockage. Further study is needed to thoroughly ascertain the mechanism behind it.

The 5-Fu-DASNP complex had significant antitumor effect in vivo. At least three reasons are therefore apparent: (1) the classical therapeutic impact of 5-Fu on human breast cancer through $G_{0} / G_{1}$ phase blockage [28]; (2) the potential antineoplastic activity of the DASNP carrier material; and (3) the sustained release function of the 5-Fu complex.

In summary, DASNP is a potential new drug carrier for cancer chemotherapy. The drug-sustaining release profile of

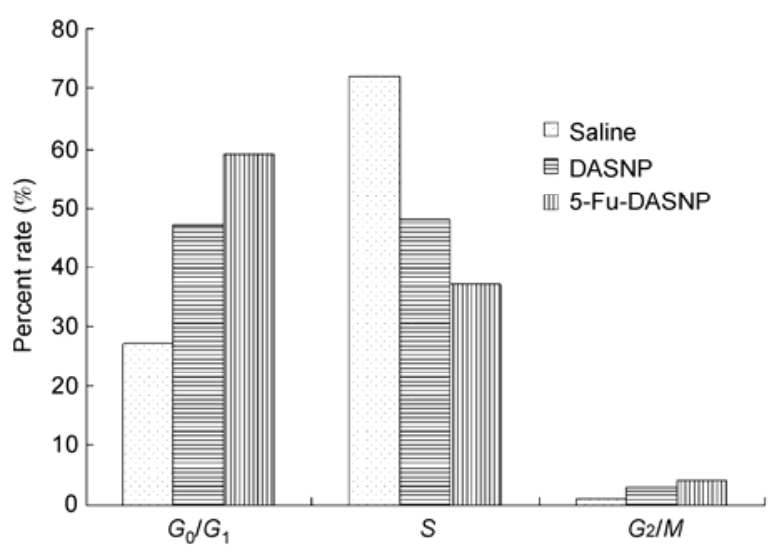

Figure 9 The influences of nanoparticles in every phase of cell cycle in human breast cancer.
DASNP for 5-Fu likely contributes to its pronounced antitumor activity. This feature may be useful in similar treatments in humans. Hence, DASNP as a non-toxic carrier of antitumor drugs must be further explored.

This work was supported by the National Natural Science Foundation of China (31100433).

1 He X, Shen B, Liu X. Production and applications on dialdehyde starch (in Chinese). Chin J Bioprocess Eng, 2004, 2: 1-4

2 Wongsagona R, Shobsngobb S, Varavinita S. Preparation and physicochemical properties of dialdehyde tapioca starch. Starch Starke, 2005, 57: 166-172

3 Para A, Karolczyk-Kostuch S. Metal complexes of starch dialdehyde dithiosemicarbazone. Carbohydr Polym, 2002, 50: 151-158

4 Tang R, Du Y, Fan L. Dialdehyde starch-crosslinked chitosan films and their antimicrobial effects. J Polym Sci Pt B-Polym Phys, 2003, 41: 993-997

5 Wang H, Wei R, Shen H, et al. Study on flexible immobilized papain with dialdehyde Starch (in Chinese). Chin J Bioprocess Eng, 2004, 2: 25-29

6 Onishi H, Nagai T. Characterization and evaluation of dialdehyde starch as an erodible medical polymer and a drug carrier. Int J Pharm, 1986, 30: 133-141

7 Langmaier F, Mokrejs P. Hydrogels of collagen hydrolysate crosslinked with dialdehyde starch. J Therm Anal Calorim, 2009, 98: 807-812

8 Song Y, Tu J, Zheng Q. A comparison study of wheat gluten composites filled with dialdehyde starch and native starch. J Polym Environ, 2010, 18: 260-265

9 Zhang S, Wang X, Zhang Y. Preparation of a new dialdehyde starch derivative and investigation of its thermoplastic properties. J Polym Res, 2010, 17: 439-446

10 Para A, Karolczyk-Kostuch S, Fiedorowicz M. Dihydrazone of dialdehyde starch and its metal complexes. Carbohydr Polym, 2004, 56: 187-193

11 Para A. Complexation of metal ions with dioxime of dialdehyde starch. Carbohydr Polym, 2004, 57: 277-283

12 Onishi H, Nagai T. Characterization and evaluation of dialdehyde starch as an erodible medical polymer and a drug carrier. Int J Pharm, 1986, 30: 133-141

13 Chen H G, Liu L W, Lü S, et al. Immobilization of aspergillus niger xylanase on chitosan using dialdehyde starch as a coupling agent. Appl Biochem Biotechnol, 2010, 162: 24-32

14 Yu D M, Xiao S Y, Tong C Y, et al. Dialdehyde starch nanoparticles: Preparation and application in drug carrier. Chin Sci Bull, 2007, 52: 2913-2918

15 Benbowa J W, Aubrechtb J, Banker M J, et al. Predicting safety toleration of pharmaceutical chemical leads: Cytotoxicity correlations to exploratory toxicity studies. Toxicol Lett, 2010, 197: 175-182

16 Prakasa Babu P, Yoshida Y, Su M, et al. Immunohistochemical expression of $\mathrm{Bcl}-2$, Bax and cytochrome following focal cerebral ischemia and effect of hypothermia in rat. Neurosci Lett, 2000, 291: 196-200

17 Hanafy S M, Shehata O H, Farahat N M. Expression of apoptotic markers Bcl-2 and Bax in chronic hepatitis $\mathrm{C}$ virus patients. Clin Biochem, 2010, 43: 1112-1117

18 Azevedo A M, Martins V C, Prazeres D M, et al. Horseradish peroxidase: A valuable tool in biotechnology. Biotechnol Annu Rev, 2003, 9: 199-247

19 Smith A A. Specifc staining of tissue components with metal-hematoxylin complexes. Micron, 2002, 33: 95-103

20 Veelaert S, Wit D D, Gotlibt K F, et al. Chemical and physical transitions of periodate oxidized potato starch in water. Carbohydr Polym, 1997, 33: 153-162 
21 Para A, Karolczyk-Kostuch S, Hajdon T, et al. Dialdehyde starch of low degree of oxidation and its derivatives. Pol J Food Nutr Sci, 2000, 50: 7-12

22 Cao S, Frank C, Shirasaka T, et al. 5-Fluorouracil prodrug: Role of anabolic and catabolic pathway modulation in therapy of colorectal cancer. Clin Cancer Res, 1995, 1: 839-845

23 Daumar P, Decombat C, Chezal J M, et al. Design, synthesis and in vitro drug release investigation of new potential 5-Fu prodrugs. Eur $\mathrm{J}$ Med Chem, 2011, 46: 2867-2879

24 Fournier E, Passirani C, Colin N. Development of novel 5-Fu-loaded poly (methylllidene malonate)-based microspheres for the treatment of brain cancers. Eur J Pharm Biopharm, 2004, 57: 189-197
25 Lamprecht A, Yamamoto H, Takeuchi H, et al. Microsphere design for the colonic delivery of 5-fluorouracil. J Control Release, 2003, 90: 313-322

26 Marija G D, Emilija F K, Katerina G, et al. 5-Fluorouracil in topical liposome gels for anticancer treatment-Formulation and evaluation. Acta Pharm, 2003, 53: 241-250

27 Para A, Ropek D. Starch dialdehyde derivatives as novel complexions protecting entomopathogenic nematodes from heavy metals. Chemiai Inz Ekol, 2000, 7: 1213-1220

28 Han W, Chen Y, Liu W. Effects of chemotherapeutic drugs on proliferation of tongue carcinoma cells (in Chinese). J Jilin Univ, 2006, 32: $672-674$

Open Access This article is distributed under the terms of the Creative Commons Attribution License which permits any use, distribution, and reproduction in any medium, provided the original author(s) and source are credited. 